\title{
Dissipative and Damping Properties of Multi-layered Rubber-Metal Vibration Absorber
}

\author{
Vladimirs Gonca ${ }^{1}$, Svetlana Polukoshko ${ }^{2}$, Egons Lavendelis ${ }^{1}$ \\ ${ }^{1}$ Riga Technical University, Institute of Mechanics. \\ Address: 6, Ezermalas str, LV-460, Riga, Latvia \\ ${ }^{2}$ Ventspils University College, Engineering Research Institute "VSRC". \\ Address: 101a, Inzenieru str, LV-3601, Ventspils, Latvia
}

\begin{abstract}
Rubber and rubber-metal (RM) elements are successfully used as bearing, joints, compensating devices, vibration and shock absorbers in civil engineering and machine building because of rubber and rubberlike materials (elastomers) have a capability of absorbing input energy much better than other construction materials. The elastic properties of rubber in such supports allows reverse backward to its original position under dynamic load action. Along with the instantaneous elastic deformation these materials exhibit a retarded elastic deformation, viscous flow (creep) and relaxation. The mechanical properties of rubber which are necessary for the optimal design of antivibration devices are next: bulk modulus of compression, dynamic and static shear modulus, energy dissipation factor. To describe the relationship between the compressive (or shear) stress $\sigma(t)$ and strain $\varepsilon(t)$ the creep and relaxation kernel, taking into account the viscoelastic properties of the rubber, is used. The kernels proposed by $A$. Rzhanitsin, Y. Rabotnov, M. Koltunov give satisfactory results for the mechanical properties of rubber in the mean frequency domain $\left(10^{-3}<\omega<10^{3} \mathrm{~s}^{-1}\right)$. In this paper for the accounting of dissipative properties of the rubber Rabotnov's kernel is used, the energy loss during one oscillation period is calculated. The flat-type RM absorber with kinematic excitation, which lower base oscillates harmonically is considered, oscillation parameters of the upper base on which the protected object is placed, are calculated. Damping properties are expressed by the ratio of the amplitude of the forced oscillations of the upper base (and object) to the amplitude of driving lower base.
\end{abstract}

Keywords: vibration damping, relaxation kernel, dynamic shear and compression moduli, rubber-metal package.

\section{INTRODUCTION}

Nowadays under conditions of intensification of production processes, increasing of the equipment capacity and high-speed vehicles creation the question of preventing the harmful effects of vibration requires special attention. In the most cases it is necessary to provide of shock and vibration protection devices at the design stage [1] - [5].

Elastomers (rubber and rubber-like materials) are the most suitable material for anti-vibration devices due to its adhesive characteristics, the ability to acquire a predetermined shape at press processing after curing, high elasticity and high internal damping. The elastic properties of rubber characterized by a large difference between bulk and shear moduli $(\mathrm{K} / \mathrm{G}$ ratio reaches $500 \div 5000)[3],[6]-[10]$. The high elasticity of rubbers allows them to withstand the large elongation without breaking. Consequently, the rubber has a greater capacity to store energy per unit volume compared with other materials.
Rubber and rubber-metal parts (rubber and metal connected by vulcanization or gluing) are used as elastic supports of veicle and machinery and are designed to reduce the dynamic loads of the working machines on their foundations or floors of buildings or for the protection of equipment and devices from bumps, shock and vibration loading operating on their base. The packages of thin-layered rubber-metal elements (rubber layer widh/height ratio $>20$ ) are also successfully used. Thin-layered rubber-metal elements (TRME) - essentially anisotropic elastic element made of rubber (or other elastomeric) and metal (or hard plastic) assembled in a package of two or more layers having a high load capacity (more than $30 \mathrm{MPa}$ in the normal to layer direction) and higher compliance (50 ... $200 \%$ from relative deformation) in the transverse direction [9] - [13].

Rubber is a material that has a clearly defined relaxation properties, so its mechanical properties strongly depend on the time of application of external loads, that is, the mode of deformation [10], [14] - 
[18]. If the duration of the force causing a deformation equal to the time of relaxation processes in the rubber, the elastic deformation is fully manifested, and this mode of deformation will correspond to well-defined modulus of rubber.

When rate of deforming load application increases modulus of elasticity will be changing, and its magnitude will be more than elastic modulus for the mode with full implementation of the elastic process.

Since many rubber-metal products serve as elastic links experience an impulsive load, alternating-sign load, periodical and non-periodical high-frequency load, it is necessary to to take into account the characteristics of the mechanical properties of rubber under dynamic loads [9] - [13].

In this paper the vibration absorber operating under uniaxial deformation (compression or shear) is considered. In Fig. 1 the scheme of mounting of this device are shown: RM absorber with flat layers (cylindrical or prismatic form) is placed between protected object and base. Rubber layers 1 are reinforced by perfectly rigid undeformable plates 2 , which is cured on to the elastomers by vulcanizing.

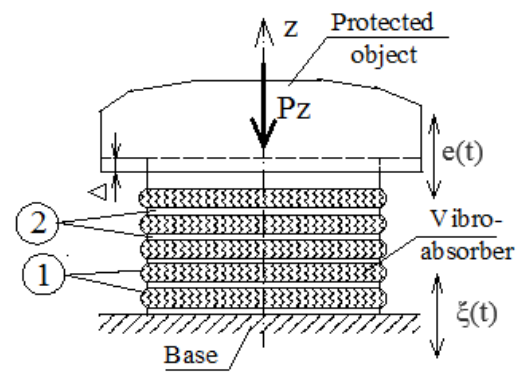

(a)

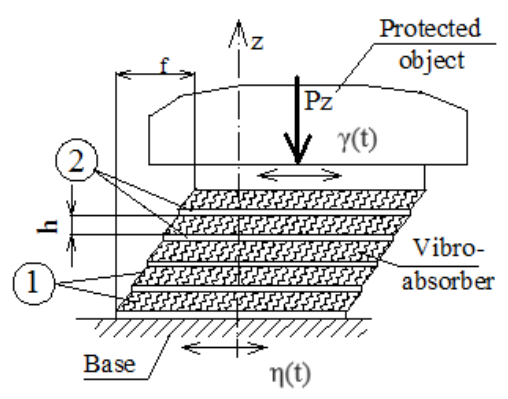

(b)

Fig. 1. Scheme of the installed absorbers: (a) anti-vertical vibrations, (b) anti-horizontal vibrations; 1-rubber layers of height $h, 2$-metal layer.

The aim of this work is to determine the dependence of this absorber dissipative and damping properties on the viscoelastic behaviour of elastomer, on the forced vibration frequency and natural frequency of the system for the model with one degree of freedom (ODF) under the action of harmonic excitation. Mass of isolator is not taken into account because of its smallness. The damping properties are determined by the ratio of the amplitude of the upper plate forced oscillations to the amplitude of the forcing oscillation of lower plate. Dissipative properties of vibroabsorber are estimated by the energy loss during one period of oscillation. Such approach allows to appoint the dimentions of antivibration devices at design stage.

\section{MATERIALS AND METHODS}

\section{Visco-elastic properties in rubber model}

In the mechanical properties of rubber a set of features manifest itself: alongside with the instantaneous elastic deformation these materials exhibit retarding elastic deformation, viscous flow (creep) and relaxation. For the description of the elastic and dissipative processes for the most materials a combination of two types of mechanical elements, elastic and viscous, may be used. Elastic element is controlled by Hooke's law, viscous - by Newton's law of flow. Connections of them in series or in parallel joint (Maxwell model or Kelvin-Voigt model) approximately describes the visco-elastic behaviour of the material [17] - [19].

The most common relationship between stress and strain may be expressed by means of integral equations of relaxation and creep. In this case accounting of the time factor influence is reduced to solving systems of integro-differential equations. Methods of solving such systems are very diverse, the most widely used methods based on the use of integral transforms, especially Laplace and Fourier transforms, and also integral operators Volterra's type [13].

In the case of axial deformation for weakly filled rubbers and small deformation $(\varepsilon \leq 20 \%)$, when the linear dependence between stress and strain exists, the dependence is valid:

$$
\sigma(t)=E_{t} \varepsilon(t)
$$

where $E_{t}$ - time operator, which does not depend on $\sigma$ and $\varepsilon$ and reflects the viscoelastic behaviour of system.

This dependence may be written in the form of an integral equation of Volterra:

$$
\sigma(t)=E_{0}\left[\varepsilon(t)-\int_{0}^{t} R(t-\tau) \varepsilon(\tau) d \tau\right]
$$

where: $E_{0}$ - instantaneous compressive modulus (or $G_{0}$ - instantaneous shear modulus), which specifies the instantaneous stiffness in the classical sense;

$R(t-\tau)$ - relaxation kernel describing the viscoelastic properties of the rubber. The presence of relaxation kernel determines the dissipative properties of rubber.

Using Fourier - images transform of $\sigma(\omega), \varepsilon(\omega)$ and $R(\omega)$, equation (1) may be written as:

$$
\sigma(\omega)=E_{0}(1-R(\omega)) \varepsilon(\omega)=E(\omega) \varepsilon(\omega)
$$

where $E(\omega)$ - - dynamic compression modulus of elasticity (it may be also $G(\omega)$ - shear modulus). 


$$
\sigma(\omega)=\int_{-\infty}^{\infty} \sigma(t) e^{-i \omega t} d t
$$

analogically for $\varepsilon(\omega), R(\omega)$.

The elastic compressive modulus $E(\omega)$ expressed as complex modulus is:

$$
E(\omega)=E^{\prime}(\omega)+i E^{\prime \prime}(\omega) .
$$

Splitting $E_{0}(1-R(\omega))$ in (2) into real and imaginary parts we obtain:

$$
\begin{aligned}
& E^{\prime}(\omega)=E_{0} \operatorname{Re}(1-R(\omega)), \\
& E^{\prime \prime}(\omega)=-E_{0} \operatorname{Im} R(\omega) .
\end{aligned}
$$

Let's denote

$$
\begin{gathered}
\operatorname{Re}(1-R(\omega))=B_{1}(\omega), \\
-\operatorname{Im} R(\omega)=B_{2}(\omega),
\end{gathered}
$$

and rewrite the elastic complex modulus $E(\omega)$ as:

$$
E(\omega)=E_{0} B_{1}(\omega)+i E_{0} B_{2}(\omega) .
$$

Proposed by A. Rzhanitsin, Y. Rabotnov and M. Koltunov kernels are in most common use. These kernels allow satisfactory describing the mechanical properties of rubber in the medium frequency range $10^{-3}<\omega<10^{3} s^{-1}$. In this work the Rabotnov's kernel is considered, which represents fractional exponential function $R(t-\tau)=\ni_{\alpha}(-\beta, t-\tau)$ :

$$
R(t-\tau)=(1-\tau)^{\alpha} \sum_{n=0}^{\infty} \frac{(-\beta)^{n}(t-\tau)^{n(1-\alpha)}}{\Gamma[(n+1)(1+\alpha)]},
$$

where $\Gamma$-gamma-function, $-1<\alpha<0, \quad \beta>0$.

In this equation $\alpha$ - parameter of fractionality, expressed in terms of the maximum value of the spectrum of relaxation times and modulus defect, $\beta$ rheological characteristics of rubber equal to:

$$
\beta=t_{0}^{-1-\alpha},
$$

where $t_{0}$ - generalized relaxation time.

Rheological parameters are tabulated for many types rubbers.

Fourier image for Rabotnov's kernel is:

$$
R(\omega)=\frac{1}{(i \omega)^{(i+\alpha)}+\beta} .
$$

Taking into account (3) real and imaginary parts we obtain from (5):

$$
\begin{gathered}
B_{1}(\omega)=\frac{\omega^{1+\alpha} \cos \delta+\beta}{\omega^{2(1+\alpha)}+2 \omega^{1+\alpha} \beta \cos \delta+\beta^{2}}, \\
B_{2}(\omega)=\frac{\omega^{1+\alpha} \sin \delta}{\omega^{2(1+\alpha)}+2 \omega^{1+\alpha} \beta \cos \delta+\beta^{2}}, \\
\delta=\frac{\pi}{2}(1+\alpha) .
\end{gathered}
$$

The coefficients $B_{1}$ and $B_{2}$ are the Fourier cosine transform and sinus transform of the fractional exponential function (4):

$$
\begin{aligned}
& B_{1}=\int_{0}^{\infty} R(z) \cos (\omega z) d z, \\
& B_{2}=\int_{0}^{\infty} R(z) \sin (\omega z) d z .
\end{aligned}
$$

If the deformation of the elastomer is harmonic, equation (1) may be written as:

$$
\begin{gathered}
\sigma(t)=E_{t} \varepsilon_{0} \sin (\omega t) \\
\sigma(t)=E_{0} \varepsilon_{0}\left[\sin (\omega t)-\int_{-\infty}^{t} R(t-\tau) \sin (\omega \tau) d \tau\right]= \\
=E_{0} \varepsilon_{0}\left[\left(1-B_{1}\right) \sin (\omega t)+B_{2} \cos (\omega t)\right]
\end{gathered}
$$

The coefficients $B_{1}$ and $B_{2}$ are the same for compression and shear deformation. In the case of share:

$$
G^{\prime}(\omega)=G_{0} B_{1}(\omega) \text { and } G^{\prime \prime}(\omega)=G_{0} B_{2}(\omega) .
$$

In this case stress is calculated by the same way.

\section{Model of damping system with one degree of freedom}

Most clearly it is possible to estimate the dynamic properties of the RME in the frame of the simplified one-degree-of-freedom model, which allows to obtain explicit analytical dependences for dissipation and damping characteristics of RME. Such model, presented in Fig.1, is considered below. Protected object is represented as a point mass, vibration isolator is placed between the protected object and the vibrating base with kinematic excitation in accordance with time dependence of $\xi(t)$. The time dependence of the movement of the protected object is $e(t)$.

Since $\xi$ and $e$ are defined in the inertial system, the relative motion of the object and base will be equal to $\xi(t)-e(t)$, and the deformation of compression of the elastomer layers with total thickness $h_{\Sigma}$ is equal to: 


$$
\varepsilon(t)=\frac{\xi(t)-e(t)}{h_{\Sigma}} .
$$

Let function $\xi(t)$ is periodic with amplitude $\xi_{0}$ :

$$
\xi(t)=\xi_{0} \sin (\omega t) .
$$

Since the system is linear, for the harmonic input function (e. g. deformation) all caused by its output processes (e. g. stress) will also be harmonic functions with the same frequency. Then it is natural to assume that function $e(t)$ will be as follows:

$$
e(t)=e_{0} \sin (\omega t+\varphi),
$$

where $e_{0}$ - amplitude vibrations of steady-state vibrations of mass, $\varphi$ - angle of lag.

The force imposed on the object with mass $m$ from the

TRM support is equal to:

$$
F=A \sigma(t),
$$

where $A$ - area of the upper face of the vibration.

The equation of motion of mass $m$ in the $z$ direction is:

$$
m \ddot{e}(t)=-A \sigma(t) .
$$

Let us find the mass displacementdepending on the viscoelastic properties of the rubber.

Taking into account (1) we have:

$$
m \ddot{e}(t)=-A E_{0}\left[\varepsilon(t)-\int_{-\infty}^{t} R(t-\tau) \varepsilon(\tau) d \tau\right]
$$

Let denote the natural frequency of vibrating mass as $\omega_{0}$, taking into account (8):

$$
\omega_{0}=\sqrt{\frac{A E_{0}}{h_{\Sigma} m}}
$$

Substituting (8), (9) and (10) into (11) and taking into account (12) we received the equation of the object motion in the form:

$$
\begin{aligned}
& \ddot{e}(t)-\omega_{0}{ }^{2}\left[(\xi(t)-e(t))-\int_{-\infty}^{t} R(t-\tau)(\xi(\tau)-e(t)) d \tau\right]=0 \\
& \text { or: } \\
& \ddot{e}(t)+\omega_{0}{ }^{2} e_{0} \sin (\omega t+\varphi)-\omega_{0}{ }^{2} \xi_{0} \sin (\omega t)- \\
& -\omega_{0}^{2}\left[\int_{-\infty}^{t} R(t-\tau)(\xi \sin (\omega \tau)-e \sin (\omega \tau+\varphi)) d \tau\right]=0
\end{aligned}
$$

For harmonic deformation taking into account linear independency of the functions $\sin (\omega t)$ and $\cos (\omega t)$, using the property $R(t-\tau)=0$ if $t<\tau$, the system of the equations is obtained from (13):

$$
\left|\begin{array}{cc}
\cos (\varphi) & -\sin (\varphi) \\
\sin (\varphi) & \cos (\varphi)
\end{array}\right| \cdot\left|\begin{array}{c}
\left(1-B_{1}(\omega)\right)-\frac{\omega^{2}}{\omega_{0}^{2}} \\
B_{2}(\omega)
\end{array}\right|=\frac{\xi_{0}}{e_{0}}\left|\begin{array}{c}
\left(1-B_{1}(\omega)\right) \\
B_{2}(\omega)
\end{array}\right|
$$

From system (14) we can define the amplitude of the forced vibration $e_{0}$ of mass and the angle of lag $\varphi$ :

$$
\begin{gathered}
\frac{e_{0}}{\xi_{0}}(\omega)=\frac{B_{1}{ }^{2}-2 B_{1}+B_{2}{ }^{2}+1}{\sqrt{\left(B_{2} b^{2}\right)^{2}+\left(B_{1}{ }^{2}+B_{1} b^{2}-2 B_{1}+B_{2}{ }^{2}-b^{2}+1\right)^{2}}}, \\
\varphi(\omega)=\arctan \frac{-B_{2} b^{2}}{B_{1}{ }^{2}+B_{1} b^{2}-2 B_{1}+B_{2}{ }^{2}-b^{2}+1}, \\
b=\frac{\omega}{\omega_{0}} .
\end{gathered}
$$

In (15) and (16) $B_{1}=B_{1}(\omega), B_{2}=B_{2}(\omega)$ are the functions of excitation frequency and calculated in accordance with (6).

\section{Dissipative properties of rubber elements}

The expression (1) is the generalized Hooke's law, here $E_{0}$ defines the instantaneous stiffness of rubber in classic sense, but relaxation kernel defines the dissipative properties of rubber. Further we can calculate the work of compressive (or shear) stress per unit volume during the all-time of the existence of deformations $\varepsilon(t)$.

$$
A=\int_{-\infty}^{\infty} \sigma(t) d \varepsilon(t)=\int_{-\infty}^{\infty} \sigma(t) \dot{\varepsilon}(t) d t
$$

Expressing $\sigma(t)$ and $\varepsilon(t)$ in terms of Fourier components we receive:

$$
\int_{-\infty}^{\infty} \sigma(t) \dot{\varepsilon}(t) d t=\frac{1}{4 \pi^{2}} \int_{-\infty}^{\infty} d t \int_{-\infty}^{\infty} E\left(\omega^{\prime}\right) e^{i \omega t} d \omega^{\prime} \int_{-\infty}^{\infty} i \omega \varepsilon(\omega) e^{i \omega t} d \omega
$$

after transformation:

$$
A=\int_{-\infty}^{\infty} \sigma(t) d \varepsilon(t)=\int_{-\infty}^{\infty} \omega E^{\prime \prime}(t)|\varepsilon(\omega)|^{2} d \omega .
$$

It is seen that $\omega E^{\prime \prime}(\omega)$ defines the density of the energy dissipation due to viscoelastic rheology of rubber. This expression may be used for the estimation of the volume density of energy release. Generally speaking, at an orbitrally point of time it is impossible to divide dissipative loss and elastic energy, saved in the RM 
support. For the harmonic loading the loss of energy during the period of vibration may be calculated as:

$$
U_{T}(\omega)=\pi m \omega_{0}^{2} B_{2} b^{4} \frac{\xi_{0}^{2}}{B_{2}^{2}+\left[1-B_{1}-b^{2}\right]^{2}}, \quad b=\frac{\omega}{\omega_{0}} .
$$

Energy loss depends on natural frequency $\omega_{0}$, excitation frequency $\omega$, vibration amplitude $\xi_{0}$ of the base, as well as $B_{1}$ and $B_{2}$ coefficients. It is necessary to take into account, that $\omega_{0}$ in turn depends on vibrating mass.

This expression is necessary for attenuation factor definition, if vibration motion of the object is describe approximately, for example, by viscoelastic Maxwell model.

\section{RESULTS AND DISCUSSION}

The results of the calculation of damping properties of RM elements in accordance with above algorithm and equations are given below. Numerical examples was solved with help of Mathcad program.

In Fig. 2 plots of dependence the amplitudes ratio on excitation frequency for natural frequencies $\omega_{0}=1$, $5,10,15,20 \mathrm{~s}^{-1}$ for low-filled rubber $2959(\alpha=-0.6$ and factor $\beta=1.062$ ) are presented.

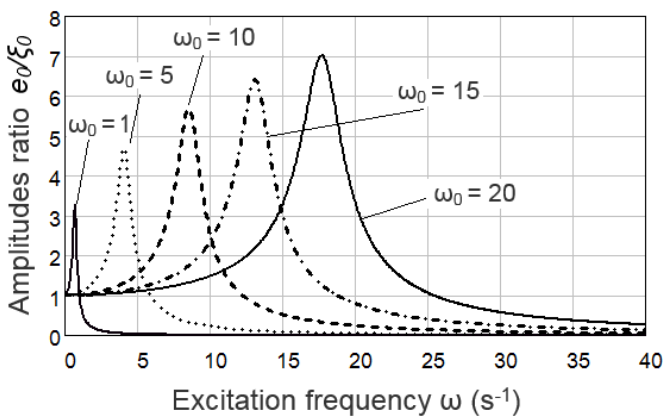

(a)

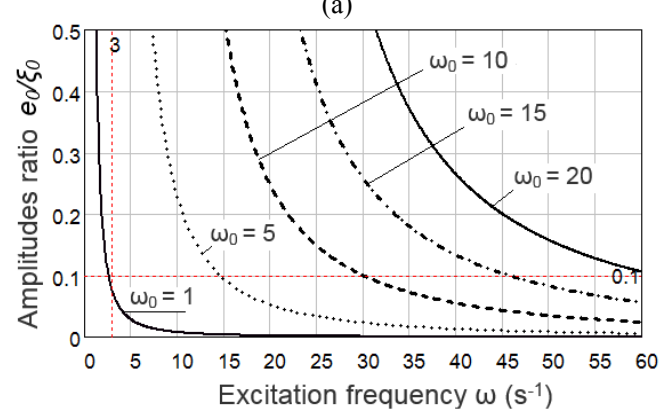

(b)

Fig. 2. Plots of dependence the amplitude ratio on excitation frequency for different natural frequencies $\omega_{0}$ and factor $\beta=1.062$.

$$
\text { (a) - full- scale plots, (b) - lower part of plots. }
$$

From Fig. 2 (b) we can see that under the excitation frequency $\omega=3,15,30,45$ and $60 s^{-1}$ the amplitude of the forced vibration of the objects becames in 10 times smaller for natural frequencies $\omega_{0}=1,5,10,15$ and $20 \mathrm{~s}^{-1}$ respectively. This confirm the well-known fact from vibration protection theory. From this graphs it is seen that RM absorber diminishes the vibration action

on the object if it's natural frequency much less than forcing frequency.

Plots of dependence the lag angle $\varphi$ on excitation frequency for different natural frequencies $\omega_{0}$ are presented in Fig. 3; factor $\beta=1.062, \alpha=-0.6$.

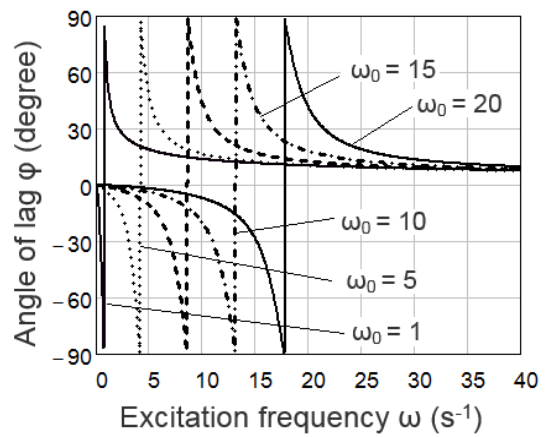

Fig. 3. Plots of dependence the lag angle $\varphi$ on excitation frequency for different natural frequencies $\omega_{0}$; factor $\beta=1.062, \alpha=-0.6$.

In Fig. 4 (a), (b) plots of dependence the amplitudes ratio on factor $\beta$ for different rubbers for natural frequencies $\omega_{0}=5 s^{-1}$ (a) $\omega_{0}=20 s^{-1}$ (b) are shown. For all rubber $\alpha=-0.6$.

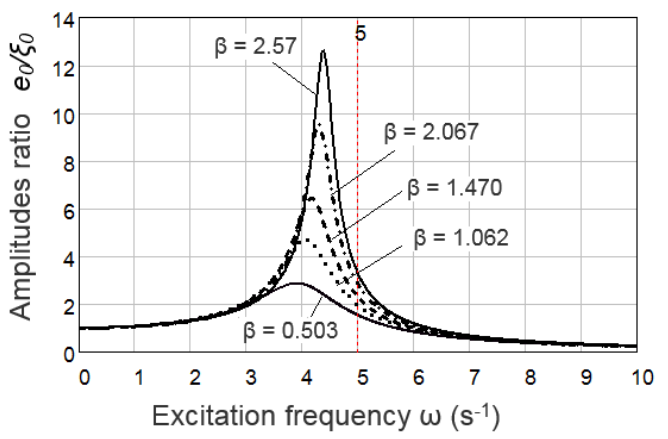

(a)

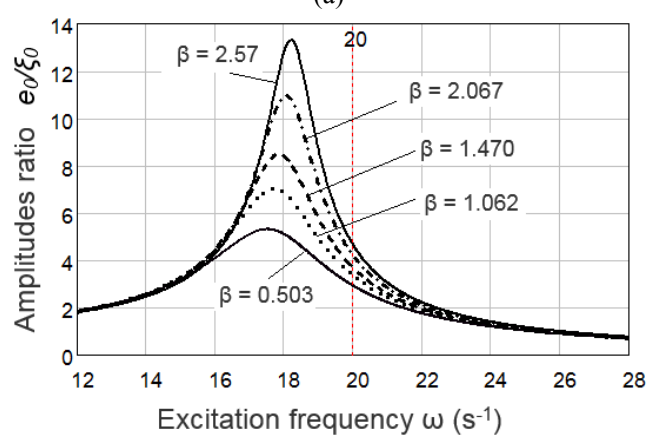

(b)

Fig. 4. Plots of dependence the amplitude ratio on excitation frequency for rubbers with $\alpha=-0.6$ and different $\beta$ factor: (a) for natural frequency $\omega_{0}=5 \mathrm{~s}^{-1}$, (b) for natural frequency $\omega_{0}=20 \mathrm{~s}^{-1}$.

Energy loss according to (18) is studied for RM absorbers with excitation amplitude $\xi_{0}=0.5 \mathrm{~mm}$, factors $\beta=1.062$ and $\alpha=-0.6$. Plots in Fig. 5 shows the dependence of energy loss per period $U_{T}$ on excitation frequency for mass $200 \mathrm{~kg}$ and $400 \mathrm{~kg}$ 
$\left(\omega_{0}=14.09 s^{-1}\right.$ and $9.96 s^{-1}$ respectively).

Reinforced RM absorber of the cylindrical form with rubber part radius $r=18 \mathrm{~mm}$ and total rubber height $h_{\Sigma}=40 \mathrm{~mm}$ is applied, $E_{0}=1.56 \cdot 10^{6} \mathrm{~N} / \mathrm{m}^{2}$.

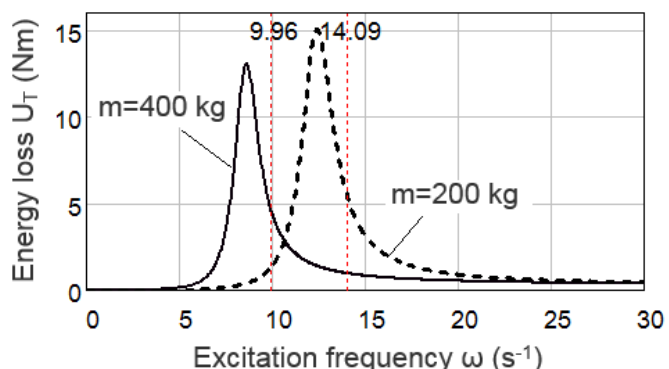

(a)

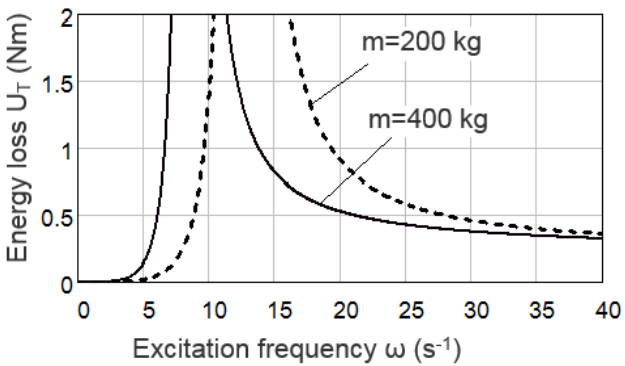

(b)

Fig. 5. Plots of dependence energy loss per period $U_{T}$ on excitation frequency: (a) for mass $200 \mathrm{~kg}\left(\omega_{0}=14.09 \mathrm{~s}^{-1}\right)$; (b) for mass $400 \mathrm{~kg}$ $\left(\omega_{0}=9.96 s^{-1}\right)$.

It is seen from the plots that the energy loss per period is identical for different mass if excitation frequency is equal to the natural frequency.

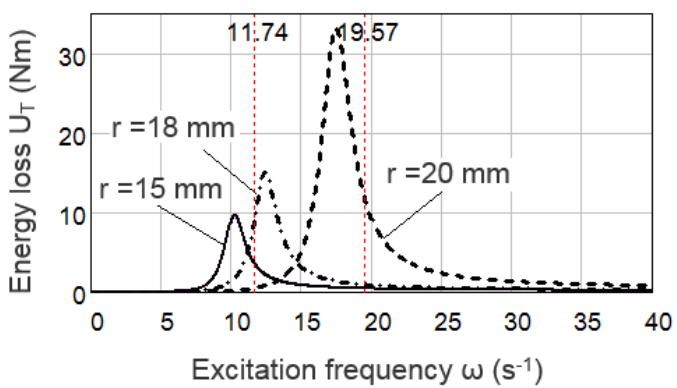

(a)

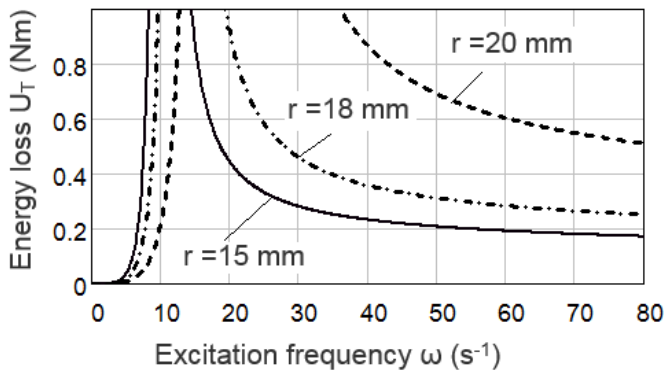

(b)

Fig. 6. Plots of dependence energy loss per period $U_{T}$ on excitation frequency for mass $200 \mathrm{~kg}$. and natural frequencies $\omega_{0}=11.74 \mathrm{~s}^{-1}$, $14.09 \mathrm{~s}^{-1}$ and $19.57 \mathrm{~s}^{-1}$
In Fig. 6 plots of the dependence of energy loss per period UT on excitation frequency for mass $200 \mathrm{~kg}$ are presented for RM absorbers with $\mathrm{h} \Sigma=40 \mathrm{~mm}$, radii 15,18 and $25 \mathrm{~mm}$ (natural frequency $\omega 0=11.74$ s-1, $14.09 \mathrm{~s}-1$ and $9.96 \mathrm{~s}-1$ respectively).

Loss of energy more distinguishes in the postresonance zone; absorbers with radius $20 \mathrm{~mm}$ deposes the energy twice as large than absorber with $\mathrm{r}=18 \mathrm{~mm}$.

\section{CONCLUSIONS}

This paper presents the procedure of the estimation of damping and dissipative properties of rubber-metal absorber devices for ODF system under harmonic exitation. Definition of damping properties is fulfilled for one degree-of-freedom system with kinematic excitation. Damping properties are expressed by the ratio of the amplitude of the forced oscillations of the upper base with the object to the amplitude of the forcing lower base oscillations. The dissipative properties are expressed by the energy loss per period of oscillation. The flat layer rubber - metal absorber of cylindrical form is calculated as the examples. Numerical examples was solved with help of Mathcad-15 program. The fulfilled calculations confirm the known fact of the theory of vibration protection: if the natural frequency of the object is three times lower than the frequency of the forcing oscillations, the forced oscillation amplitude is attenuated approximately in ten times.

It can be concluded that the proposed approach is suitable for dimensioning of RM absorber on the design stage depending on the prevented object mass, its operating mode and taking into account physical Experimental study of the dissipative and damping properties of rubber-metal vibration absorber will be the next step of the current research work mechanical properties of the elastomeric layers.

\section{REFERENCES}

[1] J. M. Kelly and D. A. Konstantinidas, Mechanics of Rubber Bearings for Seismic and Vibration Isolation. UK: John Wiley \& Sons, 2011.

[2] A. N. Gent, Engineering with Rubber: How to Design Rubber Components. Munich: Carl Hanser Verlag, 2011.

[3] J. T. Bauman, Fatigue, Stress and Strain of Rubber Components: Guide for Design Engineers. Munich: Carl Hanser Verlag, 2008.

[4] D. Konstantinidis, J. M. Kelly, Advances in Low-Cost Seismic Isolation with Rubber: Proceedings of the 10th National Conference in Earthquake Engineering, July 21-25, 2014, Earthquake Engineering Research Institute, Anchorage, AK, 2014.

[5] Lihua Zou, Kai Huang, Yu Rao, Ran Guo, Zhixu Xu, "Research on isolation property of prestressed thick rubber bearings". JVE Journal of Vibroengineering, March 2013, Volume 15, Issue 1. pp. 383-394.

[6] V. A. Lepetov, Rubber technical products. Moscow: Chemistry, 1972. (In Russian)

[7] V. T. Lyapunov, E. E. Lavendel and S. A. Shlyapochnikov, Rubber vibration isolators. Habdbook. Russia, Leningrad: Sudostrojenie, 1988. 216 p. (In Russian). 
[8] S. I. Dimnikov, Design of Rubber Elements of Structures. Latvia, Riga: Zinatne, 1991. (in Russian)

[9] E. E. Lavendel, Design of Rubber Products. Moscow: Mashinostroenie, 1976. (in Russian)

[10] V. M. Malkov, Mechanics of Multi-layered Elastomeric Structures. St.-Petersburg, Russia: St.-Petersburg University Press, 1998. (in Russian)

[11] N. S. Gusyatinskaya, Application of Thin Layer Rubber-Metal Elements in Machine-Tools and Other Engines. Moscow: Mashinostroenie, 1978. (In Russian).

[12] N. N. Frolov, S. J. Moldovanov and S. B. Lozovoy, Mechanics of Thin Rubber Elements (monograph). Kuban State Technological University, Krasnodar: Publishing House "Jug", 2011. (in Russian).

[13] N. A. Leykand, E. E. Lavendel and V. A. Hrichikova, "Calculation of Compression Rigidity of Thin-layer RubberMetal Elements." In book: Problems of Dynamics and Strength, Issue 38, pp. 57-63, Riga, 1981. (in Russian)

[14] T. O. Ormonbekov and U. T. Begaliev, Use of Thin RubberMetal Elements in Earthquake Protection of Buildings, Engineering Constructions and Equipments. Kyrgyzstan, Bishkek: Ilim, 1996.

[15] A. R. Bhuiyan, A. F. M. S. Amin, T. Hossain, Y. Okui. "Nonlinear viscosity law for rate-dependent response of high damping rubber: FE implementation and verification." Constitutive Models for Rubber $V$ - Boukamel, Laiarinandrasana, Meo \& Verron (eds), Taylor \& Francis Group, London, 2008, pp. 279 - 284.

[16] A. S. Kravchuk, A. I. Kravchuk, "Simulation on creep by hereditary theory in a simple model of constant thickness deformed coating" Electronic scientific journal "Apriori. Series: natural and technical sciences". no 2, 2014. Available: http://apriori-journal.ru/seria2/2-2014/Kravchuk-Kravchuk.pdf

[17] V. N. Poturaev and V. I. Dyrda, Rubber Machine Elements. Voscow: Mashinostrojenie, 1977.

[18] A. R. Bhuiyan, Y. Okui, H. Mitamurab, T. Imaic, "A Rheology Model of High Damping Rubber Bearings for Seismic Analysis: Identification of nonlinear viscosity", International Journal of Solids and Structures, 46, pp. 1778 1792, 2009. Available: http://www. sciencedirect. com/ science/article/pii/S0020768309000298

[19] A. F. M. S. Amin, M. S. Alam and Y. Okui, "Measurement of Lateral Deformation in Natural and High Damping Rubbers in Large Deformation Uniaxial Tests". Journal of Testing and Evaluation, Vol. 31, no 6, pp. 524-532, Nov. 2003, Available: http://www.saifulamin.info/publication/journals/j4.pdf 